\title{
Apoptosis of lymphocytes in SLE: the level, correlation with prednisolone dosage and lymphocyte phenotypes Abdelmarouf Mohieldein ${ }^{1}$, Natalia Belushkina ${ }^{2}$, Uliana PETROVA ${ }^{3}$.
}

\begin{abstract}
: -
Introduction

SLE is a systemic autoimmune disorder. One of the mechanisms by which elimination of autoreactive lymphocytes takes place is apoptosis. Adefect in apoptosis may thus contribute to the development of autoimmune disease.

Patients and methods

The study included 34 Russian children patients with SLE in age between 6-17 years old. Lymphocytes were isolated from venous blood by method of gradient centrifugation of all the blood through a Ficoll-pak solution. The quantity apoptotic cells was determined in leukocytes by flow cytometry Epics XL-2 ("Beckman Coulter", USA). Analysis of lymphocyte subpopulations was carried by using two fluorescent labels: FITC conjugated monoclonal antibodies (CD-25 and CD-20) and phycoerythrin conjugated monoclonal antibody (CD-3, CD-4 and CD-8).

Results
\end{abstract}

After in vitro incubation in $\mathrm{CO}_{2}$ incubator for $36 \mathrm{hr}$; the percentage of apoptotic lymphocytes from patients with SLE was significantly higher than from healthy donors $(7.2 * \pm 4.7 \%$ vs. $5.5 \pm 3.2 \%$ respectively). This was found to be depending upon the SLE disease activity. Moreover, observed correlations between level apoptosis lymphocyte in SLE and the titer of antibodies to dsDNA $(r=0.5)$ and dosage prednisolone $(r=0.6)$.

By analyzing lymphocyte phenotypes; there was no significant difference in percentage of CD3+T-cells from

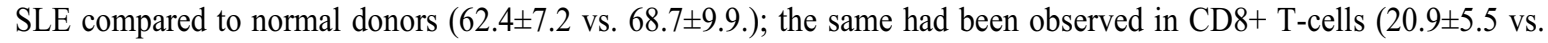
$25.3 \pm 5.6)$; and in $\mathrm{CD} 20+$ B-cells $(9.7 \pm 8.9$ vs. 13.7 \pm 8.0$)$. However, the percentage of CD4+T cell was significantly decreased $(\mathrm{P}=0,001)$ in patients with SLE compared to normal donors $\left(25.5^{*} \pm 7.3\right.$ vs. $\left.40.6 \pm 8.7\right)$. The immunoregulatory index was decreased in SLE compared to normal donors (1.2 vs 1.6 respectively). This phenotypes disbalance was associated with significant increased in the level of activated CD25+ lymphocytes in SLE compared to normal healthy donors.

Key-Words: - SLE, apoptosis, lymphocyte phenotypes, prednisolone, dsDNA autoantibodies, flow cytometry

Systemic lupus erythematosus (SLE) is a systemic autoimmune disorder with a wide spectrum of clinical and immunological abnormalities. The etiopathogensis of SLE is unknown ${ }^{1}$.

SLE characterized by polyclonal activation of B lymphocytes and the production of a wide range of antibodies to nuclear and cytoplasmic antigens.

1. Department of Biochemistry. Faculty of Medicine, Omdurman Islamic University, SUDAN.

2. Sechenov Moscow Medical Academy, Research Institute of Molecular Medicine. Moscow. RUSSIA.

3. Deparment of immunology. Speransky

Pediatrics' Hospital. Moscow. RUSSIA.

Correspondence to: Dr. Abdelmarouf H.

Mohieldein

Department of Biochemistry, Omdurman Islamic University. P. O. Box 11414. Khartoum11111, Sudan.

E-mail: mabdelmarouf@hotmail.com
The persistence of autoreactive $\mathrm{B}$ and $\mathrm{T}$ lymphocytes is thought to be responsible for hypergamma-globulinaemia and autoantibody production in SLE ${ }^{2,3}$.

One of the mechanisms by which elimination of autoreactive lymphocytes takes place is programmed cell death (apoptosis) and a defect in apoptosis may thus contribute to the development of autoimmune diseases ${ }^{4}$. A possible role of apoptotic cells in the pathogenesis of SLE has been suggested ${ }^{5}$. Moreover, it has been demonstrated that during the apoptotic process autoantigens are exposed at the outer surface of the apoptotic cell. This might allow the development of autoantibodies directed to intracellular localized antigens ${ }^{6}$.

\section{Patients and methods}

The characteristic of patients

The study included 34 children patients with SLE in age between 6-17 years old. The diagnosis and stages of SLE were established by criteria of American Rheumatism Association 
based on clinical, immunological, and instrumental methods of research.

Isolation of mononuclear and polymorph-onuclear leukocytes from human peripheral blood

Lymphocytes were isolated from venous blood by method of gradient centrifugation of all the blood through a Ficoll-pak solution (density $=1,077 \mathrm{~g} / \mathrm{ml}$ ). Granulocytes were received from the fraction of cells settled at the bottom of the test-tube, of the centrifuged blood through Ficoll gradient centrifugation. Granulocytes purified from erythrocytes by osmotic shock method in icy water-bath.

Determination quantity of apoptotic leukocytes

The quantity apoptotic cells was determined both in fresh intact peripheral blood leukocytes, and after incubation in RPMI-1640 culture medium with addition of penicillin of 50 I.U. $/ \mathrm{ml} ; 50 \mathrm{mg} / \mathrm{ml}$ of streptomycin; $10 \%$ PBS $(\mathrm{pH} 7,4)$ and $2 \mathrm{mM}$ glutamine in $\mathrm{CO}_{2}$ incubator at $5 \% \mathrm{CO} 2$ for $36 \mathrm{hr}$.

Cells fixed in $70 \%$ ethanol for $1 \mathrm{hr}$. at $+4 \mathrm{C}^{0}$; then resuspended in $1 \mathrm{ml}$ DNA-staining reagent $(\mathrm{PBS}, \mathrm{pH} 7.4$, containing $0.1 \%$ of triton X-100. 0,1mM EDTA (pH 7.4), $0.05 \mathrm{mg} / \mathrm{ml}$ ribonuclease $\mathrm{A}$, and $50 \mathrm{mg} / \mathrm{ml}$ propidium iodide) for $1 \mathrm{hr}$. at room temperature. The stained cells analyzed by flow cytometry Epics XL-2 ("Beckman Coulter", USA). Apoptotic nuclei were found out as wide peak of hypodiploid DNA which were easily distinct from narrow diploid peak DNA of normal cells.

\section{Analysis of lymphocyte subsets}

The analysis of lymphocyte subpopulations carried out by flow cytometry the analyzer and the sorter of cells EPICS XL-2 ("Beckman coulter", USA). For excitation of fluorescence used the argon laser with a wave length excitation of $488 \mathrm{~nm}$. Suspended lymphocytes incubated with monoclonal antibodies from Sorbent company (Russia). We used two fluorescent labels: FITC (fluorescein isothiocyanate) conjugated monoclonal antibodies (CD-25 and CD-20) and phycoerythrin (a red luminescence) conjugated monoclonal antibody (CD-3, CD-4 and CD-8).

\section{Determination quantity of apoptotic T-lymphocyte subsets}

$1 \times 10^{6}$ Cells of the general lymphocyte subsets suspended in $50 \mu$ icy solution (PBS with $0.1 \%$ sodium azide), then added on $2 \mu$ of the required FITC-conjugated monoclonal antibody (anti-CD3, anti-CD-4, anti-CD-8, anti-CD-20), then incubate in darkness at $+4 \mathrm{C}^{\circ}$ for $30 \mathrm{~min}$.
Cells fixed in $70 \%$ ethanol and incubated at $+4 \mathrm{C}^{\circ}$ for 1 hour; $0.1 \mathrm{ml}$ propidium iodides added followed by incubation incubate at room temperature for 1 hour. Analysis of apoptotic cells carried out by flow cytometer Epics XL-2 ("Beckman Coulter", USA).

\section{Results}

Apoptosis of lymphocytes and granulocytes

The percentage apoptosis in freshly intact lymphocytes from patients with SLE and healthy donors $(1.6 \pm 1.5 \%$ vs. $1.1 \pm 0.51 \%$ respectively) are not significantly different (Fig.1).

However, after incubation $36 \mathrm{hr}$ in $5 \%$ $\mathrm{CO}_{2}$ incubator; the percentage of apoptotic lymphocytes from patients with SLE was significantly higher than from healthy donors $\left(7.2^{*} \pm 4.7 \%\right.$ vs. $5.5 \pm 3.2 \%$ respectively). On the other hand, percentages of apoptosis in granulocytes from patients with SLE and healthy donors both in freshly intact $(3.8 \pm 3.1 \%$ vs. $3.6 \pm$ $1.4 \%$ respectively), and after being cultured in culturing medium at $37 \mathrm{C}^{0}$ and $5 \% \mathrm{CO}_{2}(14.1 \pm$ $8.4 \%$ vs. $15.3 \pm 5.6 \%$ respectively); no significant difference was reveal in level of apoptosis (Fig.1).

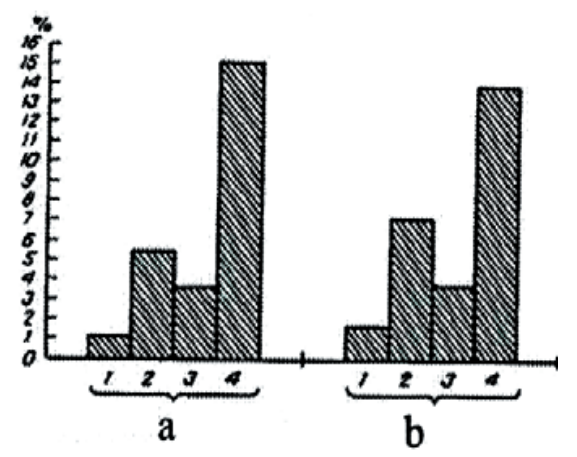

Fig.1. Percentage apoptosis lymphocytes and granulocytes freshly intact and after in vitro incubation for $36 \mathrm{hr}$, received from healthy donors (a), and patients with SLE (b). 1 - Freshly isolated lymphocytes, 2- lymphocytes after $36 \mathrm{hr}$. incubation, 3- Freshly isolated granulocytes, 4granulocytes after $36 \mathrm{hr}$. incubation.

Typical histogram of DNA distribution, freshly intact and after 18 hours incubation of lymphocytes and granulocytes fro mhealthy donors and patients with SLE, received by the flow cytometry method, is demonstrated in Fig. 2. 


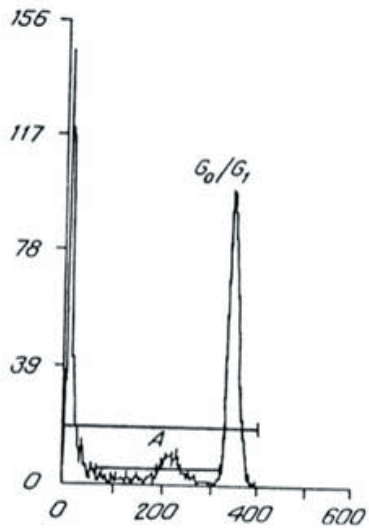

Fig. 2. Histogram illustrated the distribution of DNA lymphocytes. X-axis: the intensity of fluorescence; while Y-axis - the number of cells emitting fluorescence. Nuclei from non-apoptotic cells show a narrow peak with high fluorescence intensity, representing cells with normal chromatin content (G0 or G1 phase of cell cycle), hypodiploid - reflects quantity of apoptotic cells (A) i.e., nuclei of apoptotic cells stained in lower intensity by Propidium iodide, and can be detected in a broad peak with lower fluorescence intensity.

\section{Apoptosis of lymphocytes and SLE disease activity}

Depending upon the SLE disease activity, the level apoptosis of lymphocytes after in vitro culturing was raised (Table 1). The third stage of SLE activity was characterized by the highest level of apoptosis in lymphocytes. However, observed difference in the level apoptosis granulocytes after in vitro culturing depending similarly upon the disease activity was less expressed.

\begin{tabular}{|c|c|c|c|c|}
\hline \multirow[t]{2}{*}{$\begin{array}{c}\text { stages } \\
\text { activity } \\
\text { of SLE }\end{array}$} & \multicolumn{2}{|c|}{$\begin{array}{l}\text { \% apoptosis } \\
\text { lymphocytes } \\
\qquad(x+\text { SD) }\end{array}$} & \multicolumn{2}{|c|}{$\begin{array}{c}\text { \% apoptosis granulocytes } \\
\qquad(x+\mathrm{SD})\end{array}$} \\
\hline & $\begin{array}{l}\text { Fresh } \\
\text { intact }\end{array}$ & $\begin{array}{l}\text { After } \\
18 \mathrm{hr} \text {. } \\
\text { incubation }\end{array}$ & $\begin{array}{l}\text { Fresh } \\
\text { intact }\end{array}$ & $\begin{array}{l}\text { After } \\
18 \mathrm{hr} \text {. } \\
\text { incubation }\end{array}$ \\
\hline$I(n=7)$ & $1.5 \pm 1.1$ & $4.3 \pm 1.5$ & $3.01 \pm 1.6$ & $10.0+2.8$ \\
\hline II $(\mathrm{n}=6)$ & $1.5 \pm 0.3$ & $7.8^{*} \pm 2.0$ & $2.72 \pm 1.9$ & $12.8 \pm 3.6$ \\
\hline III $(n=6)$ & $1.1 \pm 0.11$ & $8.08^{*} \pm 1.0$ & $2.03 \pm 0.7$ & $17.3 \pm 6.8$ \\
\hline
\end{tabular}

Table 1. Dependence of the level apoptosis lympho-cytes and granulocytes upon the activity of SLE. The third stage of SLE disease activity characterized by the highest level of apoptosis in lymphocytes, while in granulocytes no significant difference observed with SLE disease activity.

\section{Apoptosis of lymphocyte subsets}

Moreover, level apoptosis was determined in such lymphocyte subsets using flow cytometry method. Our data shows that significant increased in percentage apoptosis in lymphocyte subsets CD4, CD8, and CD20 lymphocyte in patients with SLE compared to healthy donors; however, CD4 $\mathrm{T}$ cell in SLE underwent the much apoptosis than other lymphocyte subsets (Table 2).

\begin{tabular}{|c|c|c|c|c|}
\hline \multirow{2}{*}{$\begin{array}{c}\text { Group } \\
\text { investiga } \\
\text { ted }\end{array}$} & \multicolumn{4}{|c|}{ \% apoptotic lymphocytes of various } \\
& \multicolumn{3}{|c|}{ lymphocyte subpopulations $(\mathrm{X} \pm$ SD) } \\
\cline { 2 - 5 } & CD3 & CD4 & CD8 & CD20 \\
\hline $\begin{array}{c}\text { Healthy } \\
\text { donors } \\
(\mathrm{n}=22)\end{array}$ & $\mathbf{8 . 9 \pm 2 . 7}$ & $\mathbf{1 0 . 8} \pm \mathbf{3 . 5}$ & $\mathbf{1 0 . 4 \pm 2 .}$ & $\mathbf{8 . 2} \pm 2.8$ \\
\hline $\begin{array}{c}\text { SLE } \\
(\mathrm{n}=19)\end{array}$ & $\mathbf{1 7 . 5 ^ { * } \pm 8 . 6}$ & $\mathbf{2 4 . 3 ^ { * } \pm 5 . 7}$ & $\mathbf{2 0 . 5 ^ { * } \pm 8 . 5}$ & $\mathbf{1 7 . 4 ^ { * } \pm 4 . 4}$ \\
\hline
\end{tabular}

Table 2. Estimation apoptosis in various lymphocyte subsets. CD4Tcells underwent much more apoptosis in SLE tha $\mathrm{n}$ other lymphocyte subsets.

\section{Apoptosis of lymphocytes and titer of antibodies to dsDNA}

The increased level apoptosis lymphocyte in SLE was found to be correlated with the titer of antibodies to dsDNA after in vitro incubation for 36 hours. The coefficient factor of the correlations was 0.5 (Fig. 3).

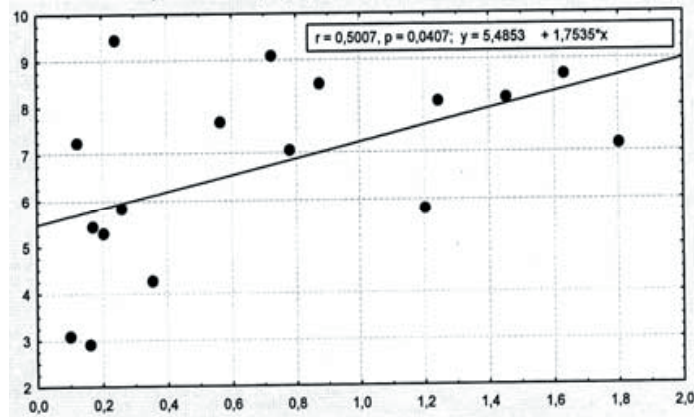

Fig.3. Correlation between percentage apoptotic lymphocytes 'after incubation in vitro for 36hr.' and the titer antibodies to dsDNA $(r=0.50)$. $X$ axis- titers antibodies to dsDNA, Y axis percentage apoptotic lymphocytes in patients with SLE after in vitro incubation for $36 \mathrm{hr}$. 


\section{Effect of prednisolone on apoptosis}

We analyzed level apoptosis in lymphocytes depending on a daily dosage prednisolone. The investigated patients with SLE received prednisolone in a daily doze from $10 \mathrm{mg}$ to $40 \mathrm{mg}$. Observed correlation of dependence ( $\mathrm{r}$ $=0,6)$ between the level apoptosis in lymphocytes and dosage prednisolone (Fig.4), expressed by the linear equation of regression.

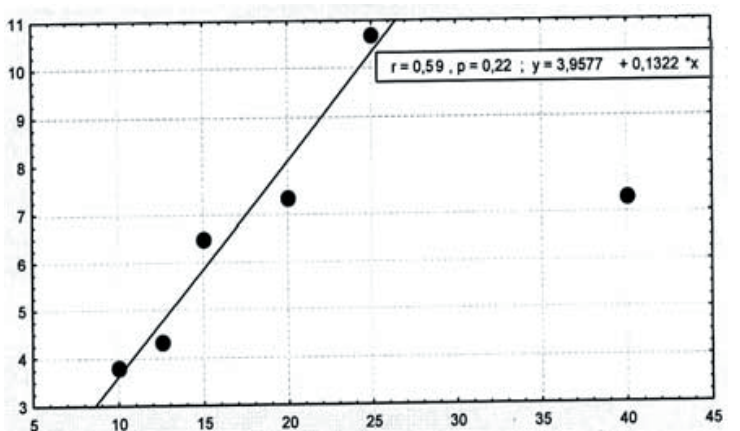

F Fig.4. The Dependence. $(r=0.6)$ of the level apoptosis in lymphocytes upon dosage prednisolone. $\mathrm{X}$ axis - Dosage prednisolone (10$40 \mathrm{mg}), \quad \mathrm{Y}$ axis - percentage apoptotic lymphocytes of patients with SLE after incubation $36 \mathrm{hr}$. $(\mathrm{r}=0.6)$.

Lymphocyte phenotypes in SLE

Our results showed that, there was no significant difference in percentage of $\mathrm{CD}^{+} \mathrm{T}$ cells from SLE compared to normal donors (62.4 \pm 7.2 vs. $68.7 \pm 9.9$.); the same observation had been seen in $\mathrm{CD} 8^{+}$T-cells $(20.9 \pm 5.5$ vs.

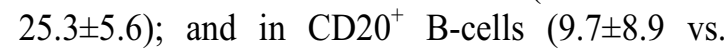
13.7 \pm 8.0$)$. However, the percentage of $\mathrm{CD} 4^{+} \mathrm{T}$ cell was significantly decreased $(\mathrm{P}=0,001)$ in patients with SLE compared to normal donors $\left(25.5^{*} \pm 7.3\right.$ vs. 40.6 \pm 8.7 ) (Fig.5).
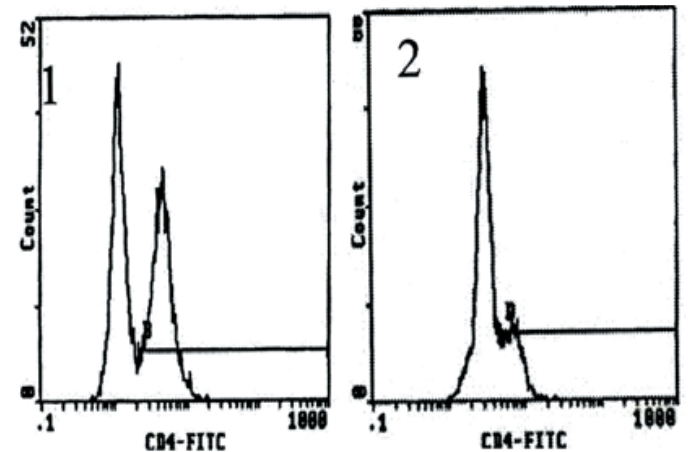

Fig.5. Analysis of lymphocyte subpopulations in patients with SLE and healthy donors. $\mathrm{X}$ axisintensity of fluorescence, $\mathrm{Y}$ axis- number of cells. Histograms, №1. CD4 ${ }^{+} \mathrm{T}$ - cells from healthy donors, № 2. $\mathrm{CD}^{+} \mathrm{T}$ - cells from patients with SLE.
The immuno-regulatory index (i.e. the ratio CD4/CD8) was decreased in SLE compared to normal donors (1.2 vs 1.6 respectively). (Fig.6). This phenotypes disbalance may be associated with change in the level of activated lymphocytes $\mathrm{CD} 25^{+}$(interleukin 2 receptor) cells, which was found to be higher in SLE compared to normal donors $\left(17.31^{*} \pm 6.8\right.$ vs. $7.44 \pm 1.0$ respectively).

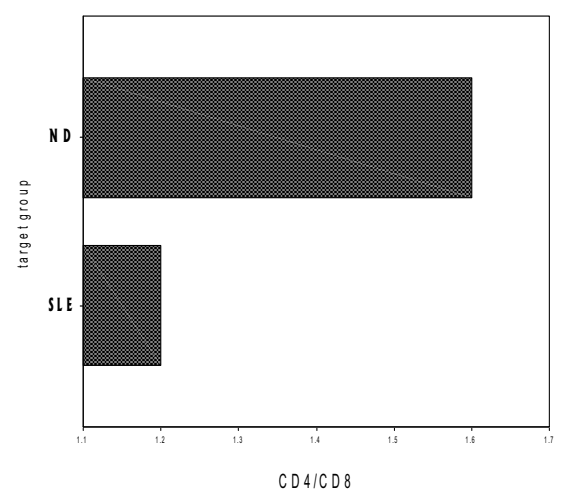

Fig.6. The immuno-regulatory index (CD4/CD8) in patients with SLE compared to healthy donors (1.2 vs. 1.6 respectively).

\section{Discussion}

The absence of statistically significant difference of lymphocytes underwent apoptosis, in freshly intact samples of peripheral blood of investigated SLE patients compared to healthy donors (fig.1), can be explained by that in peripheral blood apoptotic cells are quickly phagoctosed by macrophages that do not allow registering this phenomenon. Moreover, it was possible in this phenomenon the inhibitory effect of soluble sFas in vivo.

The significantly increased rate of apoptotic SLE lymphocytes after in vitro culturing compared to healthy donors $(7.2 * \pm 4.7 \%$ vs. $5.5 \pm 3.2 \%$ respectively) (see fig.1) may reflect an increased rate of in vivo apoptosis that reflects the in vivo activation of SLE peripheral $\mathrm{T}$ cells. Probably, apoptosis lymphocytes is a determining factor in the pathogenesis of SLE as distinguished from apoptosis granulocytes which its increase is determined by accompany inflammatory syndrome. This result is matching with the same results reported by Elmen W. et al., J. Immunol. - 1994.

Although the third stage of SLE activity was characterized by the highest level of apoptosis in lymphocytes, no significant difference in apoptosis granulocytes depending upon disease activity (table. 1). Probably, apoptosis lymphocytes is a determining factor in the pathogenesis of SLE as distinguished 
from apoptosis granulocytes which its increase is determined by accompany inflammatory syndrome. This in contrary to work done by Courtney PA et al., (Ann Rheum Dis. - 1999) who reported increased apoptotic peripheral blood neutrophils in systemic lupus erythematosus: relations with disease activity, antibodies to double stranded DNA, and neutropenia

The correlation of increased level apoptosis lymphocyte in SLE with the titer of antibodies to dsDNA after in vitro incubation for 36 hours (fig 3) is very important, because apoptosis process is the multistage process determined by a many Factors. In other words, the increased level apoptosis lymphocyte in SLE may exceed capacity of phagocytic system which may result in uncompleted apoptosis and to release of various intracellular elements, such as apoptosis specific-DNA fragments, histone proteins... etc., which promotes formation autoantibodies. This increased apoptosis of lymphocytes in SLE patients can be considered as a source of autoantigens targeted by B-cells for formation of autoantibodies which is one of SLE disease manifestation.

The observed correlation of dependence ( $\mathrm{r}$ $=0,6$ ) between the level apoptosis in lymphocytes and dosage prednisolone, expressed by the linear equation of regression (fig.4); may imply that apoptosis can be facilitated and initiated by the use of immunosuppressive medication ${ }^{7,8}$. Prednisolone may inhibit the entry of cells into G1 phase and arrest the progression of activated lymphocytes from the $\mathrm{G} 1$ to the $\mathrm{S}$ phase. This will promote apoptosis of cells and these findings confirm the data given in literatures ${ }^{9,10}$.

Since our results showed that increased apoptosis of lymphocytes after in vitro culturing, we therefore analyzed lymphocyte phenotypes in patients with SLE compared to normal healthy donors. The percentage of $\mathrm{CD}^{+} \mathrm{T}$ cell was significantly decreased $(\mathrm{P}=0,001)$ in patients with SLE compared to normal donors $(25.5 * \pm 7.3$ vs. 40.6 \pm 8.7 ) (Fig.5). The immuno-regulatory index (i.e. the ratio CD4/CD8) was decreased in SLE compared to normal donors (1.2 vs 1.6 respectively) (Fig.6).

\section{Conclusion}

As it is known that, the B-cell hyperactivity in SLE could depend on the cell activation, in particular, circulating $\mathrm{CD}^{+}$cells $^{11}$ i.e., $\mathrm{CD} 4^{+} \mathrm{T}$ cells upon interaction with naïve $\mathrm{B}$ cells triggering its proliferation and antibody production. However, our study showed significantly decrease $(P=0,001)$ in percentage of $\mathrm{CD}^{+} \mathrm{T}$ cell in patients with SLE compare to normal healthy donors $(25.5 * \pm 7.3$ vs. $40.6 \pm 8.7)$. This may be as a defense mechanism to protect certain organs from damage that may result from formation and deposition of immune complexes or may the etiology of SLE be as that of HIV infection. This is the breakthrough of our study which was the first time to be reported and registered in FEBS Special Meeting 2003 on Signal Transduction (3-8 July 2003., Belgium, Brussels).

\section{Acknowledgement:}

We would like to thank WSEAS Transactions on Biology and Biomedicine for permission to republish this article which was first published in Proceedings of the 2006 WSEAS Int. Conf. on Cellular \& Molecular Biology, Biophysics \& Bioengineering, Athens, Greece, July 14-16, 2006 (pp52-57).

\section{References:}

1. Herrmann M., Voll R. E., Zoller O. M., et al, Impaired Phagocytosis of apoptotic cell material by mobnocyte-derived macrophages from patients with systemic lupus erythematosus, Arthr. and Rheum., 1998; 41: 1241-1250.

2. Dean GS., Tyrrell-Price J., Crawley E et al Cytokines and systemic lupus erythematosus, Ann Rheum Dis. 2000; 59: 243-251.

3. Rose LM., Latchman DS., Isenberg DA, Apoptosis in peripheral lymphocytes in systemic lupus erythematosus: a review, British J. Rheumatol. 1997; 36: 158-163.

4. Mountz J.D., Wu J., Cheng J., et al. Autoimmune disease: A problem of defective apoptosis, Arthritis Rheum. 1994;37: 1415-1420.

5. Andrade F., Casciola-Rosen L., Rosen A, apoptosis in systemic lupus erythematosus. Clinical implications, Rheum Dis Clin North Am. 2000;26: 215-27.

6. Casciola-Rosen LA., Anhalt G., Rosen A, Autoantigens targeted in systemic lupus erythematosus are clustered in two populations of the surface structures on apoptotic keratinocytes, J Exp Med. 1994;179: 1317-30. 
7. Liles WC., Dale DC., Klebanoff SJ., Glucocorticoids inhibit apoptosis of human neutrophils, Blood. 1995;86: 3181-3188.

8. Thompson CB, Apoptosis in the pathogenesis and treatment of disease, Science. 1995; 267: 1456-1462.

9. Cohen JJ., DUK ER C. Glucocorticoid activation of a calcium- dependent endonuclease in thymocyte nuclei leads to cell death, J. Immunol. 1984;132: 38
10. Collins R. J., Harman B. V., Souvlis J. K. Effect of cycloheximide on B- chronic lymphocytic leukaemic and normal lymphocytes in vitro: induction of apoptosis, Br. J. Cancer. 1991;64: 518-5220.

11. Amoura Z., Piette JC., Chabre P., et al, Circulating plasma levels of nucleosomes in patients with systemic lupus erythematosus: correlation with serum antinucleosome antibody titres and absence of cle a association with diseases activity, Arthritis Rheum. 1997;40: 2217 2225 . 